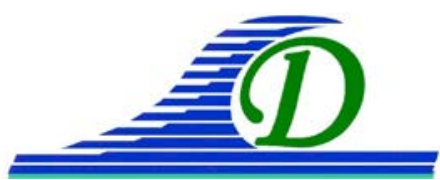

XIII ${ }^{\text {èmes }}$ Journées Nationales Génie Côtier - Génie Civil

Dunkerque, 2-4 juillet 2014

DOI:10.5150/jngcgc.2014.074 C Editions Paralia CFL

disponible en ligne - http://www.paralia.fr - available online

\title{
Balisage de protection des épaves historiques de la baie de Saint Pierre de La Martinique
}

\section{Olivier MORNET ${ }^{1}$, Sylvie GUENOT-REBIERE ${ }^{2}$ Lionel HOULLIER $^{3}$, Hervé BARREDA ${ }^{4}$}

1. Directeur de la Mer de La Martinique à Fort de France à la Direction de la Mer de La Martinique.olivier.mornet@developpement-durable.gouv.fr

2. Chargé de Mission Aménagement à Fort de France à la Préfecture de Région Martinique, Délégation à l'Aménagement du Territoire.

sylvie.guenot-rebiere@martinique.pref.gouv.fr

3. Délégué du Directeur Inter Régional de Méditerranée en Corse du Sud à Ajaccio. lionel.houllier@developpement-durable.gouv.fr

4. Responsable du Service des Phares et Balises-Polmar à Fort de France à la Direction de la Mer de La Martinique.herve.barreda@developpement-durable.gouv.fr

\section{Résumé :}

Dans le cadre du projet global d'aménagement du "Grand $S^{t}$ Pierre" à $S^{t}$ Pierre de La Martinique, destiné à renforcer la dynamique de l'offre touristique et l'équilibre des territoires, le présent article décline la mise en œuvre d'une opération visant à protéger le patrimoine culturel archéologique sous-marin que sont les épaves. Ce sont en effet 10 épaves historiques majeures qui ont coulé, le 8 mai 1902, lors de l'éruption volcanique de la Montagne Pelée. Le balisage est destiné à délimiter une zone d'interdiction de mouillage tout en offrant une zone de plongée aménagée, matérialisée par des bouées "marque spéciale" et par des coffres d'amarrage d’accueil de navires de plongée.

Dans le détail sont développés : la description des paramètres de calcul des mouillages (conditions de site et navire projet), les caractéristiques des bouées et coffres sur mouillages tendus associés, le mode opératoire chronologique de mise en œuvre, le coût et le financement de l'opération.

C'est la plus importante concentration de balisage sur mouillage tendu en grande profondeur en service (-75 m), en cela, il s'agit d'une innovation. Indiquons que ce type de balisage, respectueux de l'environnement par absence de ragage, constitue un retour d'expérience exportable.

Mots clefs : $S^{t}$ Pierre, Epave, Balisage, Mouillage Tendu, Bouée, Coffre d'amarrage, Plongée.

\section{Introduction}

C'est dans une logique de préservation des biens culturels et patrimoniaux de La Martinique que la ville de $S^{t}$ Pierre, la Région et l'État ont pris la décision de protéger les épaves reposant dans la baie de $S^{t}$ Pierre. L'espace de protection étant situé dans la 
zone des 300 mètres du rivage, la commune de $S^{t}$ Pierre, maître d'ouvrage de l'opération, accompagnée du Conseil Régional, de l'État et de l'Europe a donc mis en place la première zone d'interdiction de mouillage tout en offrant un mouillage organisé aux plongeurs loisirs. Cette zone de mouillage interdit (no anchorage) et de plongée aménagée (site for deep-sea diving) est respectivement matérialisée par 3 bouées jaunes "marque spéciale" et par 4 coffres d'amarrage d'accueil des navires de plongée. Coffres d'amarrage accessibles, pour de courte durée, à tous navires inférieurs ou égal à $13 \mathrm{~m}$ de longueur (restriction fixée par le navire projet). C'est la plus importante concentration de mouillage tendu en grande profondeur en service ( $-75 \mathrm{~m}$ pour le plus profond). Le balisage protège les épaves historiques (RORAÏMA, GABRIELLE, DIAMANT, DALHIA, THERESA, ..., voir la figure 4) coulées, le 8 mai 1902, lors de l'éruption volcanique de la Montagne Pelée (28 000 morts et 250 navires coulés). A noter que le TAMAYA, non intégré dans la zone de mouillage interdit, est de fait protégé par la grande profondeur (environ $-85 \mathrm{~m}$ ) où il se trouve. En effet, aucun navire ne mouille à ces profondeurs. De plus les plongées à l'air étant limitées à $60 \mathrm{~m}$, la présence d'une bouée à cet endroit aurait pu inciter des plongeurs à tenter une incursion "main sur main" le long d'un mouillage "sécuritaire" et potentiellement rassurant.

\section{Données relatives au projet}

\subsection{Conditions de site et nature du fond}

Soit les conditions suivantes (Sources : Météo France, instructions nautiques et cartes marines du SHOM) :

- houle max 8 à $10 \mathrm{~m}$ période 10 à $15 \mathrm{~s}$

- surcote cyclonique $0,30 \mathrm{~m}$ à $\mathrm{S}^{\mathrm{t}}$ Pierre

- vitesse du vent maxi $228 \mathrm{~km} / \mathrm{h}$

- vitesse du courant 1 à 3 nœuds respectivement dans les canaux Nord et Sud. En l'absence de donnée pour $\mathrm{S}^{\mathrm{t}}$ Pierre, on retiendra 0,5 nd dans les calculs

- hauteur maxi de la marée à St Pierre 0,60 m.

Nature du fond : Fond de sable légèrement vaseux (cendres de la Montagne Pelée).

\subsection{Navire projet}

Le navire de plongée le plus contraignant de La Martinique, pour le dimensionnement des coffres d'amarrage est "PLANETE BLEUE". Navire de $13 \mathrm{~m}$ pour $7 \mathrm{t}$.

A noter que les navires ne sont amarrés que le temps des plongées qui n’excède pas $3 \mathrm{~h}$. De plus, les plongées ont toujours lieu par beau temps. Le calcul des coffres est mené :

- par force 3 et navire amarré (houle 0,60 m, vent $19 \mathrm{~km} / \mathrm{h}$ soit 10 nd et courant 0,5 nd)

- puis avec les conditions de site énumérées ci-avant, le cas le plus défavorable en l'occurrence. 


\section{XIII ${ }^{\text {èmes }}$ Journées Nationales Génie Côtier - Génie Civil \\ Dunkerque, 2-4 juillet 2014}

\subsection{Caractéristiques du balisage et des coffres d'amarrage}

La fourniture des bouées (voir figures 1 et 2) et des coffres est détaillée ci-après, soit :

- 3 bouées jaunes "marque spéciale" type FLOATEX 1214BL-M et croix de $S^{t}$ André

- 2 bouées subsurfaces type FLOATEX Jumper 1209 de 600 litres $\left(0,6 \mathrm{~m}^{3}\right)$ de réserve de flottabilité pour les bouées : $\mathrm{n}^{\circ} 2$ et $\mathrm{n}^{\circ} 3$

- 4 coffres d'amarrage type FLOATEX MMB1290-BB blancs

- 4 bouées subsurfaces type FLOATEX Jumper 8094 de 200 litres $\left(0,2 \mathrm{~m}^{3}\right)$ de réserve de flottabilité pour les 4 coffres.

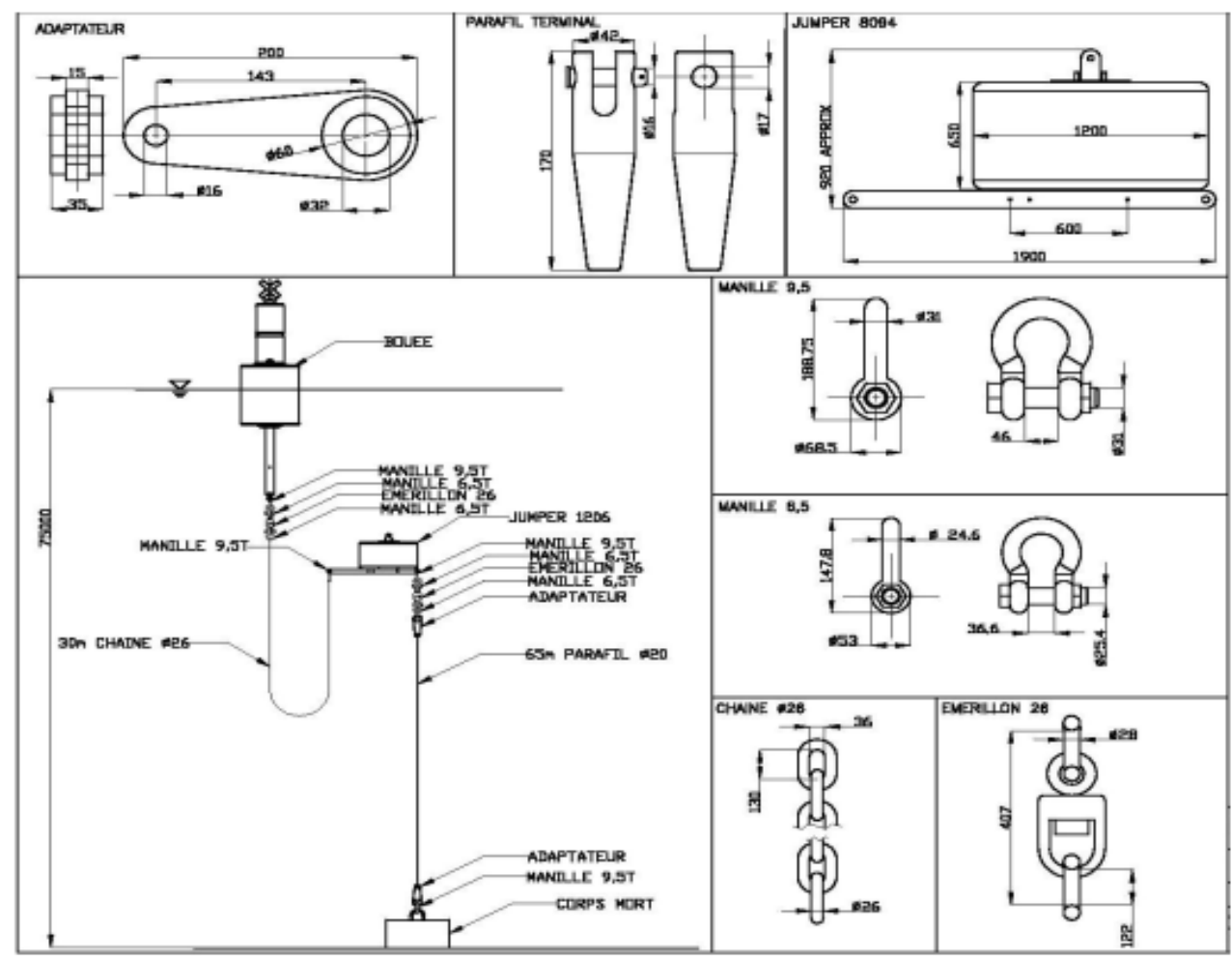

Figure 1. Plan de principe de la bouée $n^{\circ} 2(-75 \mathrm{~m})$.

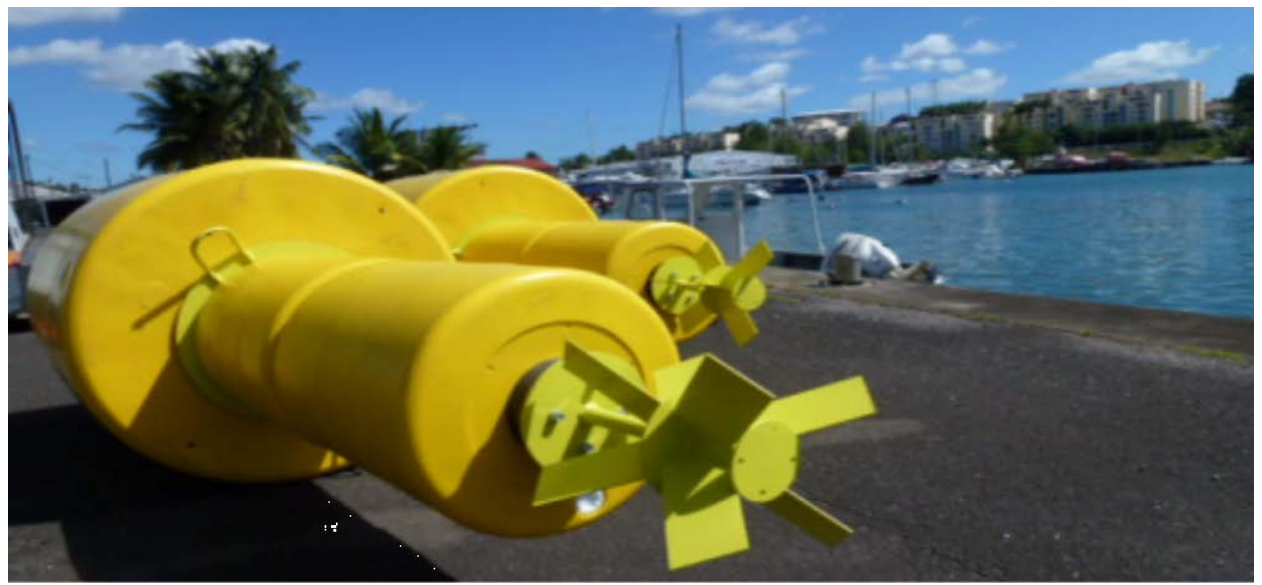

Figure 2. Bouées de marque spéciale sur le parc de balisage à Fort de France. 


\subsection{Dimensionnement du mouillage (voir le tableau 1 ci-après)}

Le dimensionnement est mené avec les logiciels GPB et Ancrage du CEREMA Direction technique Eau, mer et fleuves à Brest (Recommendation on the design of normal moorings, 1998 ; Documentation technique du CETMEF, 1997 ; Guideline n¹066 AISM, 2010). Pour un site et une bouée donnés, on calcule la longueur et le calibre du mouillage qui permettent de vérifier dans les conditions les plus défavorables que :

- le mouillage est tangent au fond (bouée $\mathrm{n}^{\circ} 1$ )

- la réserve de flottabilité, ou franc-bord, de la bouées ou du coffre est suffisant

- le coefficient de sécurité à la rupture est supérieur ou égal à 5.

Les paramètres intervenant dans les calculs sont les suivants :

- P poids de la bouée (en $\mathrm{N}$ )

- U volume de la bouée (en $\mathrm{m}^{3}$ )

- d profondeur d'eau maximale (en m)

- H Hauteur maximale de houle (en m)

- c Poids linéique déjaugé de la chaîne (en $\mathrm{N} / \mathrm{ml}$ )

- T Tension de rupture de la chaîne (en N)

La condition "mouillage tangent au fond" est satisfaite lorsque la longueur L du mouillage est supérieure à la longueur "soulevée " $L_{s}$ suivante (1) :

$$
L_{s}=\left(d+\frac{H}{2}\right) \cdot \sqrt{1+\frac{2 F}{c(d+H / 2)}}
$$

La condition "réserve de flottabilité" $R$ s'exprime en fonction de la longueur de chaîne :

$$
R=U-\frac{\left(P+L_{s} c\right)}{\rho_{w} g}
$$

La condition "réserve de flottabilité" doit permettre de s'assurer que le franc-bord est suffisant pour supporter les opérations de maintenance et de submersion. Pour le cas du mouillage tendu, la longueur est calculée de manière à ce que la bouée ou le coffre aient une réserve de flottabilité de $0,3 \mathrm{~m}^{3}$ au minimum.

La condition "résistance à la rupture" se vérifie si l'inégalité suivante est satisfaite (3) :

$$
\sqrt{F^{2}+\left(c \cdot L_{s}\right)^{2}} \leqslant \frac{T}{5}
$$

Le corps-mort doit résister aux efforts de la ligne de mouillage par frottement sur le fond, soit l'équation (4) pour calculer sa masse $M$ (en $\mathrm{kg}$ ) :

$$
M=k \frac{F \delta}{g(\delta-\rho w) \operatorname{tg}(\phi)}
$$

où : $k$ et le coefficient de sécurité au glissement (en général 1.5), $\delta$ la densité du corpsmort (en $\mathrm{kg} / \mathrm{m}^{3}$ ) et $\phi$ l'angle de frottement entre le sol et le corps-mort (en général $45^{\circ}$ ). L'utilisation d'un flotteur subsurface permet de réduire la longueur du mouillage et du rayon d'évitage et d'augmenter la réserve de flottabilité, mais en contrepartie cela 


\section{XIII ${ }^{\text {èmes }}$ Journées Nationales Génie Côtier - Génie Civil \\ Dunkerque, 2-4 juillet 2014}

augmente la masse du corps mort. Le dimensionnement proposé a été vérifié et accepté par le Titulaire du marché.

Tableau 1. Récapitulatif des caractéristiques des lignes de mouillage.

\begin{tabular}{|c|c|c|c|c|c|c|c|c|}
\hline Désignation & $\begin{array}{l}\text { Hauteur } \\
\text { d'eau } \\
(m)\end{array}$ & $\begin{array}{l}\text { Longueur } \\
\text { mouillage } \\
(m)\end{array}$ & $\begin{array}{l}\text { Mouillage } \\
\text { tendu } \\
\text { ( } m \text { et } \varnothing)\end{array}$ & $\begin{array}{l}\text { Cul de bouée } \\
\text { (m, calibre et } \\
\text { pas) }\end{array}$ & $\begin{array}{l}\text { Dormante } \\
\text { ( } m \text {, calibre } \\
\text { et pas) }\end{array}$ & $\begin{array}{l}\text { Manilles + } \\
\text { émerillons } \\
\text { (Ø) }\end{array}$ & $\begin{array}{l}\text { Flotteur } \\
\text { subsuface } \\
\text { (litres) }\end{array}$ & $\begin{array}{l}\text { Corps } \\
\text { mort } \\
(t)\end{array}$ \\
\hline Bouée $n^{\circ} 1$ & 20 & $\begin{array}{l}45 \text { m de } \\
\text { chaîne } \\
\text { (mouillage } \\
\text { classique) }\end{array}$ & 0 & 15 en $25-5 D$ & $\begin{array}{l}30 \text { en } 25- \\
5 D\end{array}$ & $\begin{array}{l}4 \text { manilles }+1 \\
\text { émerillon } \\
(25 \mathrm{~mm})\end{array}$ & 0 & 1,7 \\
\hline Bouée $n^{\circ} 2$ & 75 & $\begin{array}{l}95 \text { mixte } \\
\text { (mouillage } \\
\text { tendu) }\end{array}$ & $\begin{array}{l}65 \text { de } \\
\text { PARAFIL } \\
\text { en ø20 }\end{array}$ & 30 en $25-5 D$ & 0 & $\begin{array}{l}6 \text { manilles }+ \\
2 \text { émerillons } \\
(25 \mathrm{~mm})\end{array}$ & $\begin{array}{l}\text { JUMPER } \\
1290 \\
600 \text { litres }\end{array}$ & 2,1 \\
\hline Bouée $n^{\circ} 3$ & 35 & $\begin{array}{l}55 \text { mixte } \\
\text { (mouillage } \\
\text { tendu) }\end{array}$ & $\begin{array}{l}25 \text { de } \\
\text { PARAFIL } \\
\text { en ø20 }\end{array}$ & 30 en $25-5 D$ & 0 & $\begin{array}{l}6 \text { manilles }+ \\
2 \text { émerillons } \\
(25 \mathrm{~mm})\end{array}$ & $\begin{array}{l}\text { JUMPER } \\
1290 \\
600 \text { litres }\end{array}$ & 2,1 \\
\hline Coffre $n^{\circ} 1$ & 20 & $\begin{array}{l}25 \text { mixte } \\
\text { (mouillage } \\
\text { tendu) }\end{array}$ & $\begin{array}{l}10 \text { de } \\
\text { PARAFIL } \\
\text { en ø20 }\end{array}$ & 15 en $20-3 D$ & 0 & $\begin{array}{l}2(20 \mathrm{~mm}) \text { et } 3 \\
(25 \mathrm{~mm})+1 \\
\text { émerillon } \\
(25 \mathrm{~mm})\end{array}$ & $\begin{array}{l}\text { JUMPER } \\
8094 \\
200 \text { litres }\end{array}$ & 1,7 \\
\hline Coffre $n^{\circ} 2$ & 30 & $\begin{array}{l}35 \text { mixte } \\
\text { (mouillage } \\
\text { tendu) }\end{array}$ & $\begin{array}{l}20 \text { de } \\
\text { PARAFIL } \\
\text { en ø20 }\end{array}$ & 15 en $20-3 D$ & 0 & $\begin{array}{l}2(20 \mathrm{~mm}) \text { et } 3 \\
(25 \mathrm{~mm})+1 \\
\text { émerillon } \\
(25 \mathrm{~mm})\end{array}$ & $\begin{array}{l}\text { JUMPER } \\
8094 \\
200 \text { litres }\end{array}$ & 1,7 \\
\hline Coffre $n^{\circ} 3$ & 50 & $\begin{array}{l}55 \text { mixte } \\
\text { (mouillage } \\
\text { tendu) }\end{array}$ & $\begin{array}{l}40 \text { de } \\
\text { PARAFIL } \\
\text { en ø20 }\end{array}$ & 15 en $20-3 D$ & 0 & $\begin{array}{l}2(20 \mathrm{~mm}) \text { et } 3 \\
(25 \mathrm{~mm})+1 \\
\text { émerillon } \\
(25 \mathrm{~mm})\end{array}$ & $\begin{array}{l}\text { JUMPER } \\
8094 \\
200 \text { litres }\end{array}$ & 1,7 \\
\hline Coffre $n^{\circ} 4$ & 40 & $\begin{array}{l}\text { 45mixte } \\
\text { (mouillage } \\
\text { tendu) }\end{array}$ & $\begin{array}{l}30 \text { de } \\
\text { PARAFIL } \\
\text { en ø20 }\end{array}$ & 15 en $20-3 D$ & 0 & $\begin{array}{l}2(20 \mathrm{~mm}) \text { et } 3 \\
(25 \mathrm{~mm})+1 \\
\text { émerillon } \\
(25 \mathrm{~mm})\end{array}$ & $\begin{array}{l}\text { JUMPER } \\
8094 \\
200 \text { litres }\end{array}$ & 1,7 \\
\hline
\end{tabular}

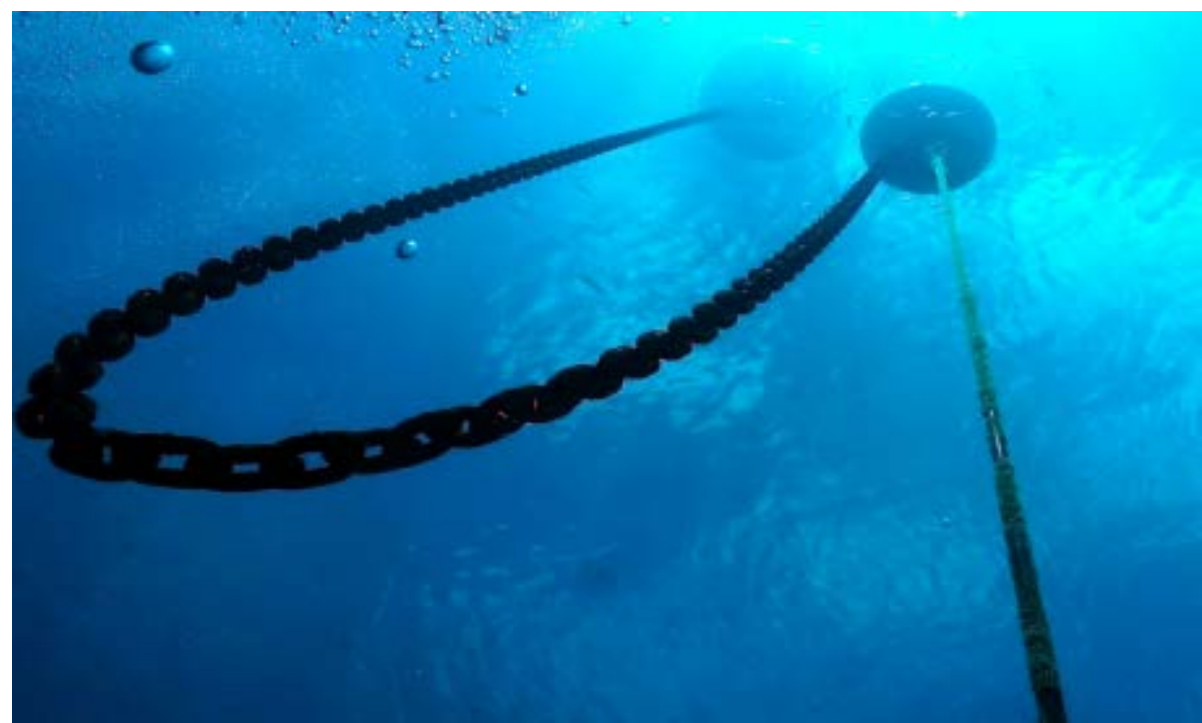

Figure 3. Vue sous-marine du mouillage tendu de la bouée subsurface, reliée au coffre 
Thème 4-Ouvrages portuaires et offshore

\subsection{Plan de situation et bathymétrie}

Les données correspondantes sont indiquées sur la figure 4 ci-après.

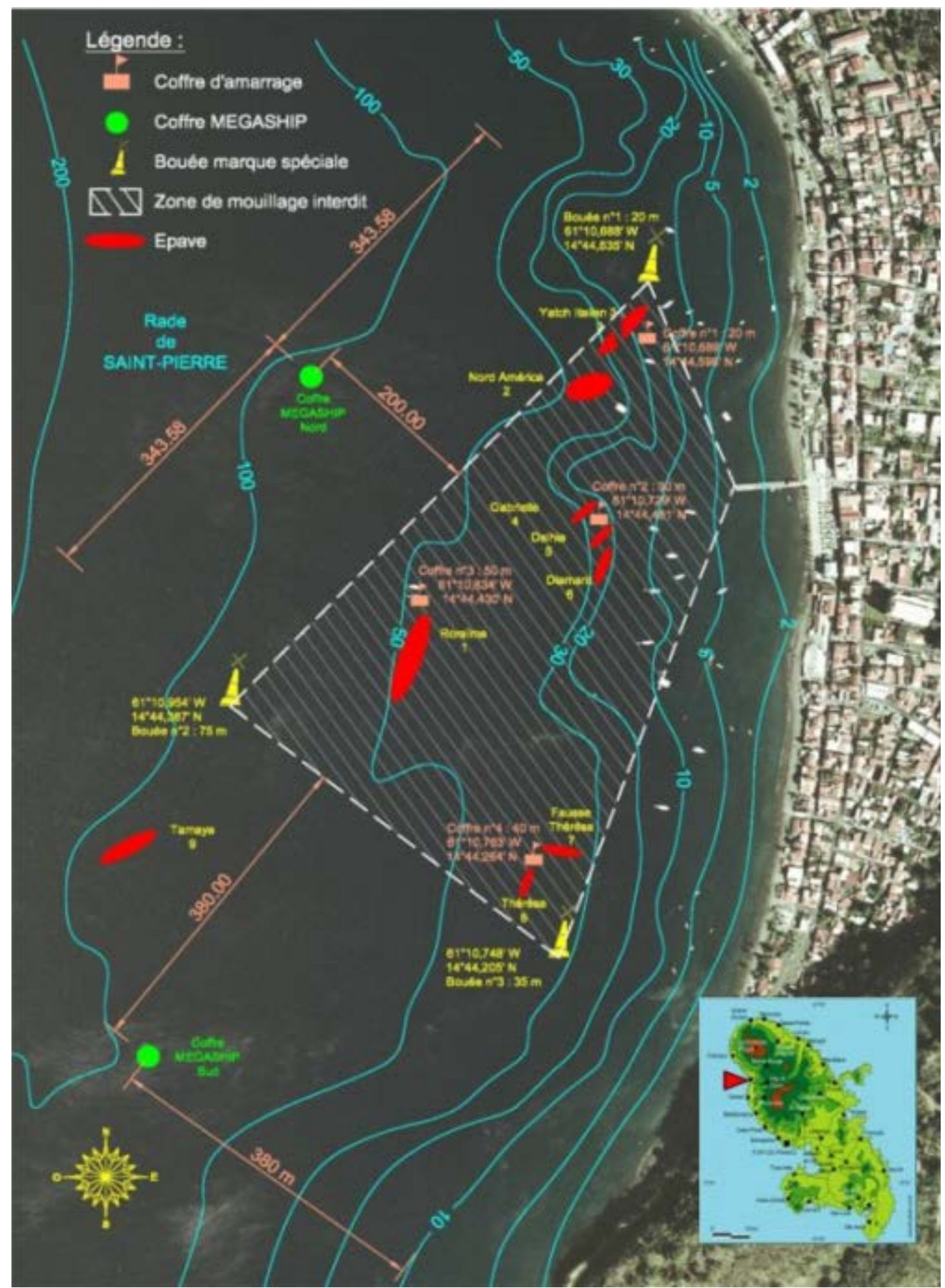

Figure 4. Extrait carte SHOM n7089 6 et Google Earth.

2.6 Positionnement des points de mouillage

Les bouées et coffres ont été placées suivants le tableau 2 


\section{XIII èmes Journées Nationales Génie Côtier - Génie Civil \\ Dunkerque, 2-4 juillet 2014}

Tableau 2. Coordonnées géographiques en WGS 84 en degrés et minutes décimales des bouées et coffres.

\begin{tabular}{lllllll}
\hline Bouée $n^{\circ} 1$ & Bouée $n^{\circ} 2$ & Bouée $n^{\circ} 3$ & Coffre $n^{\circ} 1$ & Coffre $n^{\circ} 2$ & ${\text { Coffre } n^{\circ} 3}$ & ${\text { Coffre } n^{\circ} 4}^{\circ}$ \\
\hline $61^{\circ} 10,688^{\prime} \mathrm{W}$ & $61^{\circ} 10,954^{\prime} \mathrm{W}$ & $61^{\circ} 10,748^{\prime} \mathrm{W}$ & $61^{\circ} 10,689^{\prime} \mathrm{W}$ & $61^{\circ} 10,729^{\prime} \mathrm{W}$ & $61^{\circ} 10,834 \mathrm{~W}$ & $61^{\circ} 10,763^{\prime} \mathrm{W}$ \\
$14^{\circ} 44,635^{\prime} \mathrm{N}$ & $14^{\circ} 44,367^{\prime} \mathrm{N}$ & $14^{\circ} 44,205^{\prime} \mathrm{N}$ & $14^{\circ} 44,596^{\prime} \mathrm{N}$ & $14^{\circ} 44,481^{\prime} \mathrm{N}$ & $14^{\circ} 44,430^{\prime} \mathrm{N}$ & $14^{\circ} 44,264^{\prime} \mathrm{N}$ \\
Profondeur & Profondeur & Profondeur & Profondeur & Profondeur & Profondeur & Profondeur \\
$20 \mathrm{~m}$ & $75 \mathrm{~m}$ & $35 \mathrm{~m}$ & $20 \mathrm{~m}$ & $30 \mathrm{~m}$ & $50 \mathrm{~m}$ & $40 \mathrm{~m}$ \\
\hline
\end{tabular}

\section{Mode opératoire de mise en œuvre}

\subsection{Choix du type de mouillage}

Les bouées $n^{\circ} 2$ et $n^{\circ} 3$ et les 4 coffres (voir les figures 1 et 3 ) sont sur mouillage tendu (câble $\varnothing 20 \mathrm{~mm}$ en PARAFIL pour 7,5t de tension à la rupture) avec flotteur subsurface immergé. Cette disposition est destinée à annuler le ragage sur le fond, qui aurait risqué de détruire les épaves et aussi à augmenter le franc bord par allégement du mouillage. La chaîne "frappée" sur le flotteur subsurface immergé est destinée à amortir les efforts générés par : la houle, le vent, le courant et le navire amarré (pour les coffres).

\subsection{Mouillage des bouées et coffres}

Mise en place des corps-morts des 6 mouillages tendus avec flotteurs subsurfaces associés descendus, en douceur, à l'aide du cabestan du baliseur (voir la figure 5).

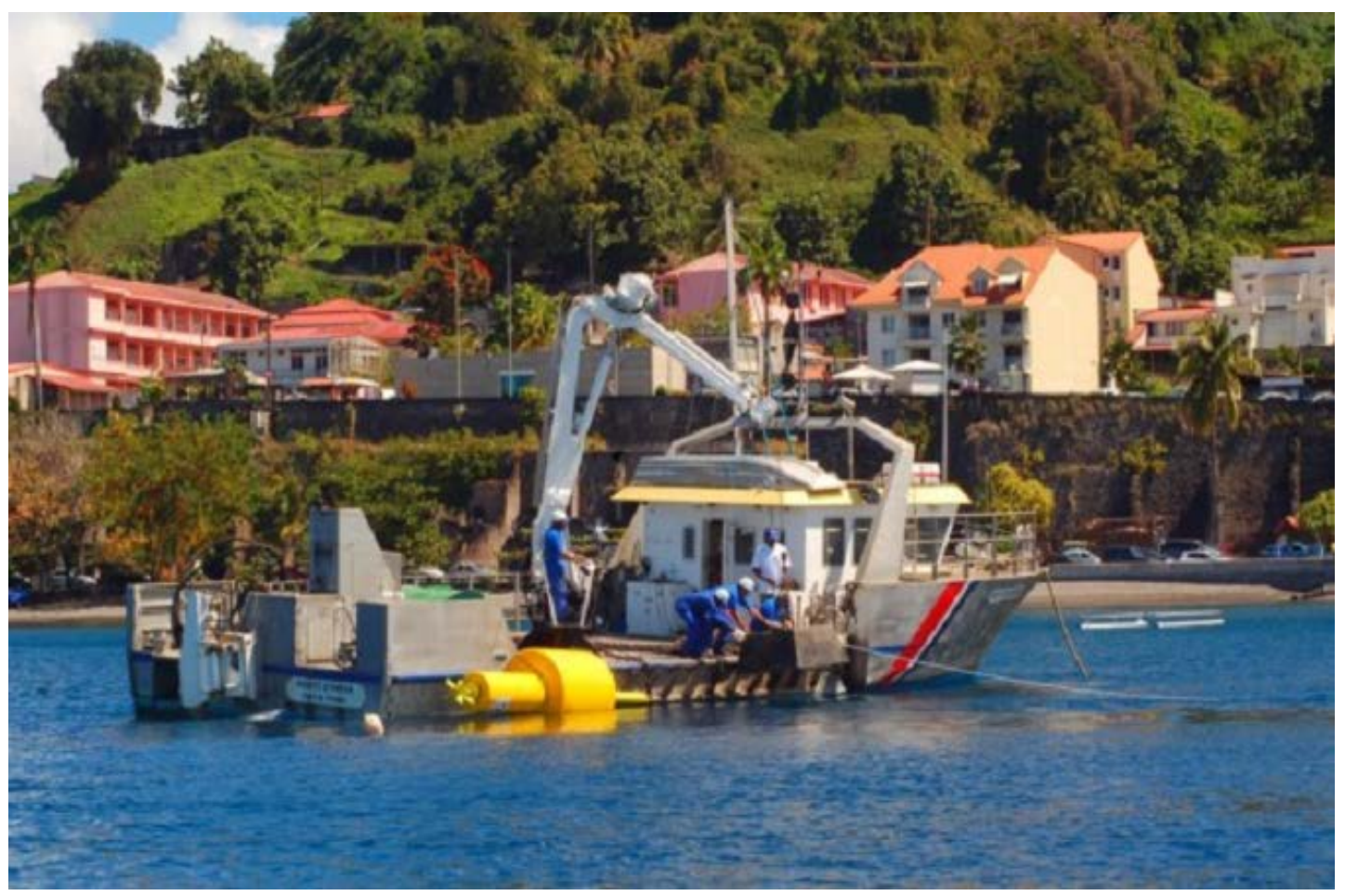

Figure 5. Baliseur "Pointe d'Enfer" en opération de mouillage. 
Les 4 corps-morts des coffres d'amarrage ont été posés sur des points préalablement identifiés par bouées signal. Précisons que les bouées signal de repérage ont été positionnées par les clubs de plongée locaux sur des points exempts d'épaves. Puis le baliseur avec son équipage renforcé par une équipe de scaphandriers ont :

- "manillé" et mouillé les bouées et les coffres sur les bouées subsurfaces

- fixé une anode sacrificielle sur chacun des corps-morts (voir la figure 6) pour se prémunir de la corrosion galvanique entre les divers métaux de l'attache (BARREDA et al., 2002 ; BARREDA \& LAZZERI, 1995 ; HACHE, 1977 ; LAMOUREUX, 1994 ; LEMAIRE, 1988).

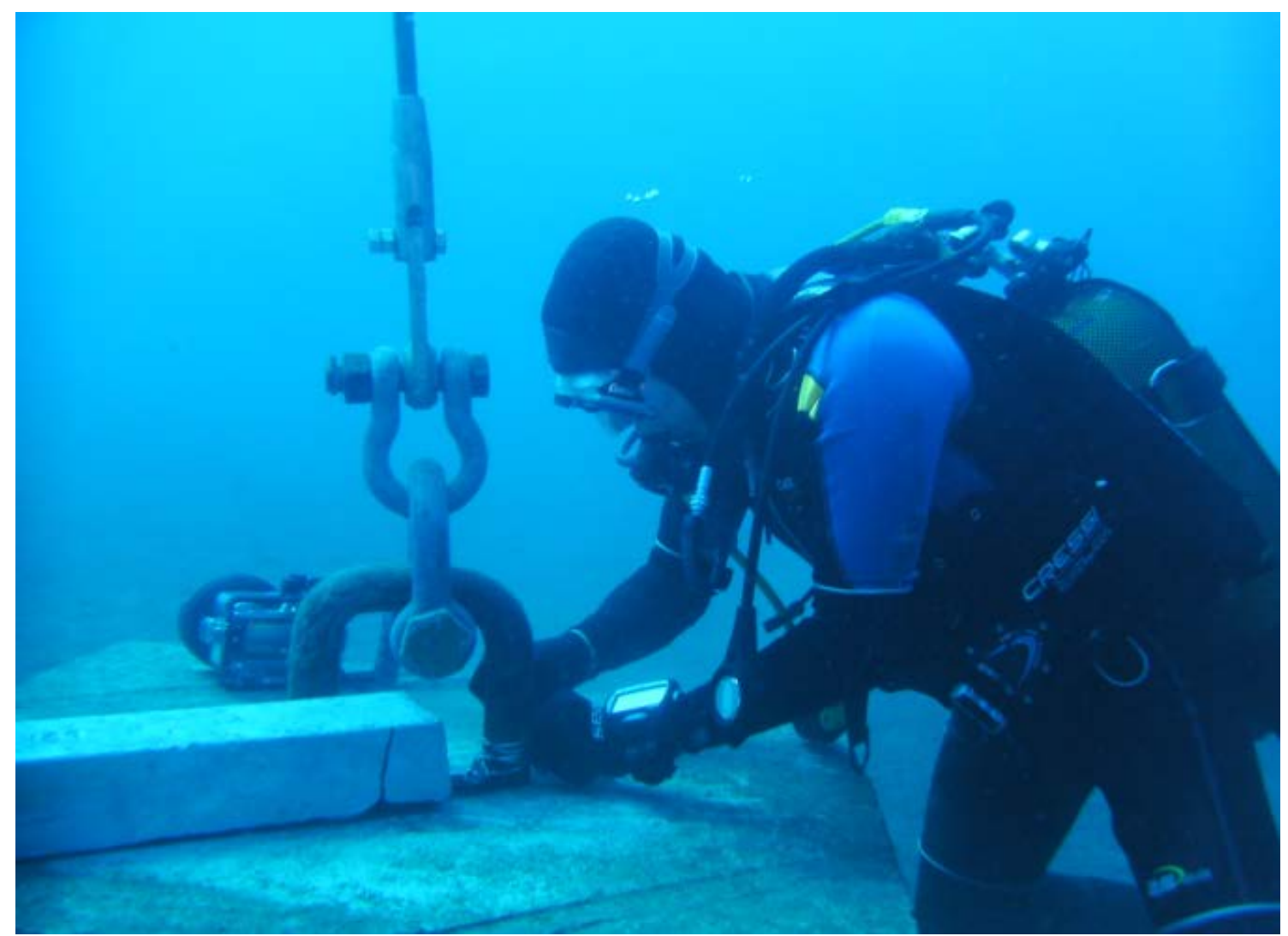

Figure 6. Mise en place d'une anode sacrificielle de $10 \mathrm{~kg}$ en aluminium à l'indium.

\section{Coût de l'opération}

L'opération s'élève à environ 60000 €TTC, soit :

- $36825 €$ correspondant au marché de fourniture (bouées, coffres, ...) et transport

- $22568 €$ suite à la convention de mise en œuvre signée entre la Mairie de $S^{t}$ Pierre et le Service des PetB-Polmar (fourniture des corps-morts, montage des bouées, des mouillages, intervention baliseur, ...).

Une convention d'entretien du balisage de 7595 €/an, a été signée avec la commune. 


\section{XIII $I^{\text {èmes }}$ Journées Nationales Génie Côtier - Génie Civil \\ Dunkerque, 2-4 juillet 2014}

\section{Financement}

Le financement étudié et mis en place par la Délégation à l'Aménagement du Territoire (DAT) de la Préfecture de région Martinique, se décline comme suit :

- Europe 44\%

- Région Martinique 22\%

- Commune de St Pierre 15\%

- DEAL (Direction de l'Environnement de l'Aménagement et du Logement) 11\%

- DAC (Direction des Affaires Culturelles) 8\%.

\section{Conclusions et perspectives}

Cette méthode de balisage qui utilise les mouillages tendus est simple à mettre en œuvre, préservatrice des fonds marins et de la flore sous-marine par l'absence de ragage. Elle présente toute les garanties de durabilité vis à vis de sollicitations météoocéaniques sévères, compte tenu des précautions et dispositions constructives qui ont été prises.

La méthode peut être utilisée indifféremment dans des mers sans marnage ou ayant un marnage moyen de 3 à 4 mètres ainsi que par petits $(-10 \mathrm{~m})$ et grands fonds $(-75 \mathrm{~m}$ et plus). En outre cette méthode de balisage constitue un retour d'expérience exportable vers d'autres pays intéressés.

\section{Références bibliographiques}

BARREDA H., LAZZERI A. (1995). Protection cathodique des palplanches en eau de mer : dimensionnement des anodes sacrificielles. Note technique du Bulletin de liaison des Ponts et Chaussées (janvier) n¹95. LCPC Paris, France.

BARREDA H., HUBERT C., FARDELLA C. (2002). Systèmes de protection anticorrosion duplex de structures métalliques à la mer. Actes du 2 colloque International d'Aix en Provence sur la protection cathodique et les revêtements associés (juin) CEFRACOR Paris, France.

Guideline $\mathrm{n}^{\circ} 1066$ (2010). On the design of floating aid to navigation moorings IALA/AISM. S ${ }^{\mathrm{t}}$ Germain en Laye, France.

HACHE A. (1977). La corrosion des métaux. Presses Universitaires de France Vendôme, France.

LAMOUREUX J.J. (1994). Précis de corrosion. Éditions Beauchemin, Laval province du Québec, Canada. 
Thème 4 - Ouvrages portuaires et offshore

LEMAIRE F. (1988). La protection contre la corrosion des structures métalliques immergées. STCPMVN, Notice STC-QG nº8-2. Compiègne, France.

Recommendation on the design of normal moorings (1998). IALA/AISM, E-107. $\mathrm{S}^{t}$ Germain en Laye, France

Signalisation maritime - Documentation technique du CETMEF - Exploitation du balisage - Lignes de mouillages (1997). CETMEF Compiègne, France. 